\title{
Estrogen Accelerates Cutaneous Wound Healing by Promoting Proliferation of Epidermal Keratinocytes via Erk/Akt Signaling Pathway
}

\author{
Tao Zhou ${ }^{\mathrm{a}}$ Zicheng Yang ${ }^{\mathrm{a}}$ Yajie Chen ${ }^{\mathrm{a}}$ Yu Chen ${ }^{\mathrm{a}}$ Zongwei Huang ${ }^{\mathrm{b}}$ Bo You \\ Yizhi Penga Jing Chen ${ }^{a}$ \\ aState Key Laboratory of Trauma, Burns and Combined Injury, Chongqing Key Laboratory for \\ Proteomics Disease, Institute of Burn Research, Southwest Hospital, the Third Military Medical \\ University, Chongqing, 'bepartment of Burn, The First Affiliated Hospital, Guangxi Medical University, \\ Nanning, People's Republic of China
}

\section{Key Words}

Estrogen $\bullet 17 \beta$-estradiol $•$ Keratinocytes $・$ Proliferation • Akt • Erk

\begin{abstract}
Background: Previous studies have established that estrogen is capable of accelerating cutaneous wound healing through multiple mechanisms, one of which involves affecting keratinocytes biological properties, such as migration, proliferation, etc. This study aims to reveal the underlying molecular mechanisms of estrogen promoting epidermal keratinocytes proliferation. Method \& Results: We found that compared with female mice with a normal estrous cycle, female mice with their ovaries removed before puberty exhibited a delayed cutaneous wound healing, thinner epidermis, and significantly fewer proliferating cell nuclear antigen (PCNA)-positive keratinocytes. Moreover, a significant increase in $\mathrm{HaCaT}$ proliferation was detected by a CCK8 assay when treated with $17 \beta$-estradiol compared with those treated with control vehicle. Consistent with the results of the CCK8 assay, flow cytometry indicated a high proportion of $17 \beta$-estradiol-treated $\mathrm{HaCaT}$ cells in S phase compared with vehicletreated cells. Western blot analysis demonstrated the activation of Akt, Erk and upregulation of PCNA in $\mathrm{HaCaT}$ cells treated with $17 \beta$-estradiol. Interestingly, Erk activation occurred prior to Akt activation. Upregulation of PCNA expression, elevated proliferation and high S phase fraction of $\mathrm{HaCaT}$ cell by $17 \beta$-estradiol could be reversed by an Akt or Erk inhibitor. Moreover, Erk inhibition reversed $17 \beta$-estradiol-induced Akt activation, whereas an Akt inhibitor exhibited no effect on Erk, further suggesting that Erk was on the upstream while Akt on the downstream of the signaling pathway. Conclusion: This study demonstrates that one of the critical mechanisms underlying $17 \beta$-estradiol promoting skin wound healing is through regulation of keratinocyte proliferation via Erk/Akt signaling pathway.

T. Zhou and Z. Yang are the first co-authors of this article.




\begin{tabular}{|c|c|c|}
\hline Cellular Physiology & Cell Physiol Biochem 2016;3 & 8:959-968 \\
\hline and Biochemistry & $\begin{array}{l}\text { DOI: 10.1159/000443048 } \\
\text { Published online: March 04, } 2016\end{array}$ & $\begin{array}{l}\text { O } 2016 \text { The Author(s). Published by S. Karger AG, Basel } \\
\text { wwwwkargercom/cp. }\end{array}$ \\
\hline
\end{tabular}

\section{Introduction}

Normal cutaneous wound healing is a complicated process involving three overlapping phases, namely the inflammatory, proliferative, and remodeling phases [1-3]. Each phase requires coordinated effects of various cell types, including keratinocytes [4, 5], fibroblasts [6], immune cells [7], etc., the disturbance of which in any phase leads to delayed cutaneous wound healing [8].

Previous studies have developed numerous methodologies to promote cutaneous wound healing [9-11], among which the application of estrogen has been widely investigated. Local utilization of exogenous estrogen accelerates the repair of acute and chronic wounds $[1,12,13]$. Estrogen plays a prominent role in promoting healing processes by modulating the inflammatory response, accelerating re-epithelialization, inducing granulation, and modifying proteolysis [14] in skin cells, especially keratinocytes [15]. The role of estrogen in promoting keratinocyte proliferation has been implicated in several previous studies. In women with low estrogen levels, skin thickness has been shown to be reduced by approximately $1 \%$ per year after menopause [16]. A study of topical estrogen administration in elderly males and females has confirmed that keratinocyte proliferation and epidermal thickness increase significantly after only two weeks of application [17]. However, the regulatory mechanisms and signaling pathway underlying estrogen-accelerated cutaneous wound healing via epidermal keratinocytes are not completely understood.

The extracellular signal-regulated kinase (Erk) and phosphatidylinositol 3-kinase (PI3kinase) signaling pathways, which have been reported to be activated by basic fibroblast growth factor (bFGF), nerve growth factor (NGF), valproic acid, etc., have been reported to be crucial for cutaneous wound healing [18-22]. Furthermore, activation of the Erk signaling pathway has been reported in keratinocyte proliferation induced by the application of exogenous estrogen [23]. However, whether estrogen can activate these pathways and subsequently regulate keratinocyte proliferation remains elusive.

Herein, we propose that estrogen accelerates cutaneous wound healing by promoting proliferation of epidermal keratinocytes via the Erk/Akt signaling pathway.

\section{Materials and Methods}

Animal model

All procedures were approved by the Animal Ethical Committee of the Third Military Medical University and were carried out in accordance with the approved guidelines. Adult female C57 mice (10 to 12 weeks of age, $20 \pm 2 \mathrm{~g}$ ) were purchased from the Center for Experimental Animals at the Third Military Medical University. The mice were maintained under appropriate conditions with food and water available ad libitum. The estrous cycle (including the proestrus, estrus, metestrus, and diestrus phases) was assessed by vaginal smear cytology according to a previously published protocol [24]. Mice in the estrus phase were chosen for this study because the systemic estrogen concentration peaks during this phase [25], and these mice were designated as the "estrus group" [26]. Bilateral ovariectomy was performed according to previous reports [27] on 4-week-old female C57 mice. The ovariectomized mice were kept until 8 to 10 weeks of age for further experiments. The two groups of mice were then anesthetized with isoflurane $(1-4 \%)$ in oxygen and shaved prior to the generation of a bilateral incision of approximately $1 \mathrm{~cm}$ in length, as described previously [18]. Photos of the incisions from the same mouse were taken at $0,1,3,5$, and 7 days following incision generation for comparison.

\section{Histological analysis}

The mice were anesthetized with isoflurane $(1-4 \%)$ in oxygen. Histological sections were prepared from wound tissues fixed in $10 \%$ buffered formalin saline and embedded in paraffin wax. Five micrometer sections were stained using a Hematoxylin Eosin (HE) staining kit (cat\#E607318-0200, BBI Life Science, New York, USA) or subjected to immunohistochemistry with a rabbit anti-PCNA antibody (cat\#D120014-0200, 1:1000, BBI Life Science, New York, USA) and an appropriate biotinylated secondary antibody, followed by detection with a GTVision ${ }^{\mathrm{TM}}+$ Detection System/Mo\&Rb (GK600705, Gene Tech, Shanghai, China) using 


\section{Cellular Physiology Cell Physiol Biochem 2016;38:959-968 \begin{tabular}{ll|l} 
and Biochemistry $\begin{array}{l}\text { DOI: 10.1159/000443048 } \\
\text { Published online: March 04, } 2016\end{array}$ & $\begin{array}{l}\text { C } 2016 \text { The Author(s). Published by S. Karger AG, Basel } \\
\text { www.karger.com/cpb }\end{array}$
\end{tabular} \\ Zhou et al.: Estrogen Promotes Keratinocyte Proliferation}

Novared substrate and counterstaining with hematoxylin. Total cell numbers were quantified using Image Pro Plus software (see Supplementary Methods online; Media Cybernetics, Silver Spring, MD).

\section{Cell proliferation assessment by CCK8 assay}

A human keratinocyte cell line (HaCaT) was purchased from the Institute of Burn Research and was cultured in serum-free medium under standard conditions as previously described [28]. HaCaT cells cultured in serum-free medium from each group were seeded on day 3 in a 96-well tissue culture plate at a density of $1 \times 10^{4}$ cells per well. Cell proliferation for each group was determined using a Cell Counting Kit (SK6335-500T, BBI Life Science, New York, USA). Absorbance was measured at $450 \mathrm{~nm}$ using an ELx800 Absorbance Microplate Reader (Bio-Tek Instruments, Winooski, VT).

\section{Cell cycle distribution analysis by flow cytometry}

Flow cytometry was performed for DNA cell cycle analysis according to the method described by Akgul [29]. Briefly, cells were treated with Hoechst 33342 (10 $\mu \mathrm{g} / \mathrm{ml}$; Sigma, Dorset, UK) and were then harvested and washed twice with phosphate-buffered saline (PBS). After the second wash, the cells were resuspended in $500 \mu \mathrm{l} \mathrm{PBS}$ containing $5 \mu \mathrm{g} / \mathrm{ml}$ propidium iodide (PI; C1052 Beyotime Institute of Biotechnology, Shanghai, China). The relative DNA concentrations in stained cells were measured using an LSR flow cytometer (Becton Dickinson, San Jose, CA, USA) and were plotted in a frequency histogram of Hoechst fluorescence. Cell cycle data were acquired using CELLQuest (BD biosciences, California, USA) and were analyzed with ModFit LTTM. DMSO-treated keratinocytes were used as control cells to identify the staining efficiency of PI and the group of diploid cells (G0/G1 phase cells). Using the G0/G1 peak of chicken erythrocyte nuclei as an internal standard, both the G0/G1 and G2/M peaks of the analyzed samples were determined, and then the DNA quantities were calculated. Cell cycle percentages were derived from the DNA histograms. The peak of hypodiploid cells, anterior to the G0/G1 peak, was the apoptosis peak. The proportions of cells in the G0/G1, $\mathrm{S}$ and G2/M phases were calculated using FlowJo software (Tree Star, Inc., Ashland, OR, USA). The S-phase fraction (SPF) was determined by measuring the area under the curve between the G0/G1 and G2/M peaks.

Determination of protein concentrations by Western blot analysis

Western blotting was performed as previously described to determine the protein expression levels of Akt, p-Akt, Erk, p-Erk, and proliferating cell nuclear antigen (PCNA) in tissues from HaCaT cells treated for $0,5,15,30,60,120$, or 240 min or 3 days [18]. In brief, proteins were homogenized in 10 volumes of RIPA lysis buffer I (C500005, Sangon Biotech, Shanghai, China); next, equal amounts of proteins (10 - 20 $\mu \mathrm{g}$ ) were fractionated on a 15\% SDS-PAGE gel and were then transferred to nitrocellulose membranes. The membranes were blocked with blocking buffer (5\% nonfat dairy milk dissolved in TBS containing $0.05 \%$ Tween-20 (TBS-T)) overnight at $4{ }^{\circ} \mathrm{C}$. They were then incubated with the following primary antibodies: antiAkt (1:1000; \#9272s, Cell Signaling Technology, Shanghai, China), anti-p-Akt (1:1000; \#4051s, Cell Signaling Technology, Shanghai, China), anti-Erk (1:1000; \#4695s, Cell Signaling Technology, Shanghai, China), antip-Erk (1:1000; \#4370s, Cell Signaling Technology, Shanghai, China), anti-PCNA (1:1000; \#AB20014, BBI Life Science, New York, USA) and anti-TUBA4A (1:500) and a secondary antibody (\#AB10058, BBI Life Science, New York, USA). Following three washes in TBS-T, the proteins were detected using an enhanced chemiluminescence (ECL; Abcam) kit according to the manufacturer's instructions.

\section{Statistics}

Statistical analysis was performed using Student's t test, one-way analysis of variance (ANOVA) and the least significant difference (LSD) test. The data are presented as the mean \pm standard deviation (SD) of four independent experiments for each group and were analyzed with GraphPad Prism (GraphPad Software, Inc., La Jolla, CA, USA). All tests were two tailed, and the level of significance was set at $\mathrm{P}<0.05$.

\section{Results}

Cutaneous wound healing was delayed in ovariectomized mice

For the mouse incisional wound healing model, the ovariectomized group exhibited significantly delayed incision healing compared with the estrus group (Fig. 1A). Wound 


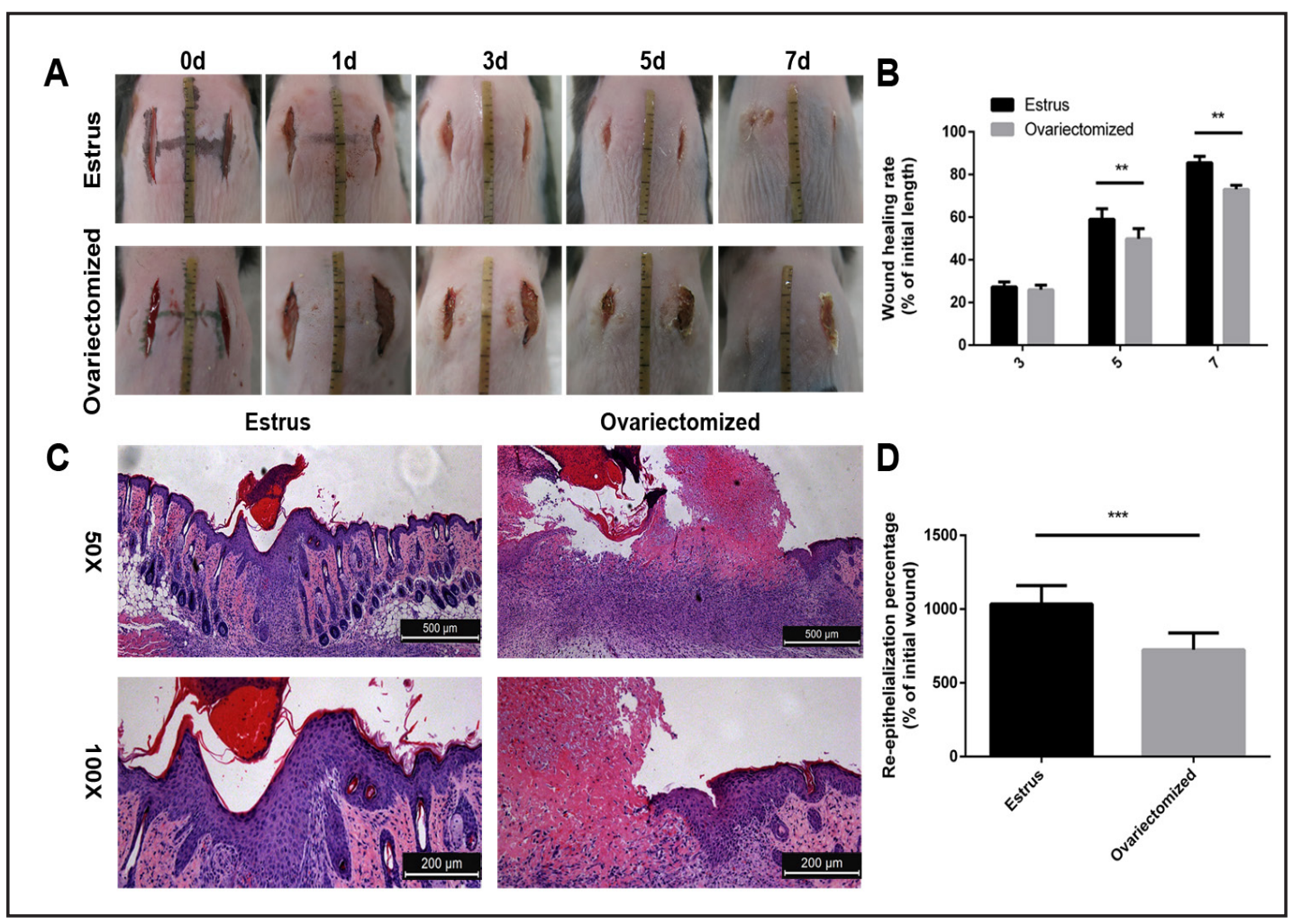

Fig. 1. Delayed cutaneous wound healing in ovariectomized mice. Representative photos of incisional wounds in mice at estrus cycle and in ovariectomized mice. (B) Statistical analysis of the healing rates (\% of initial length) of incisional wounds in the mice at estrus cycle and in the ovariectomized mice at 3, 5, or 7 days post-injury $\left({ }^{* *} p<0.01\right)$. (C) Representative HE staining images of the wounds on day 7 post-wounding. (D) Statistical analysis of the re-epithelialization percentages of incisional wounds in the mice at estrus cycle and in the ovariectomized mice at 7 days post-injury $(* * p<0.01)$.

healing progression in the ovariectomized group was similar to that in the estrus group during the first 3 days. However, accelerated healing in the mice at estrus cycle was observed compared with the ovariectomized mice thereafter, as demonstrated by the wound healing percentages, which indicated that the mice at estrus cycle exhibited better healing on days 5 and 7 than the ovariectomized mice (Fig. 1B). Representative histological images of incisions at both high and low magnifications were captured, and HE staining on day 7 after wounding demonstrated that re-epithelialization of the incision in the 5 mice at estrus cycle was complete, indicating full recovery (Fig. 1C), whereas only 2 mice in the ovariectomized group displayed complete re-epithelialization. The re-epithelialization percentages of the incisions on day 7 directly demonstrate this significant difference (Fig. 1D). Moreover, reepithelialization of the lesion sites in the mice at estrus cycle was thicker than that in the ovariectomized mice. Both the macroscopic and histological assessments demonstrated that cutaneous wound healing was delayed in the ovariectomized mice.

Keratinocyte proliferation was increased in the presence of estrogen in vivo and in vitro

As shown in Fig. 2A, the epidermal thickness of the ovariectomized mouse tail skin was significantly decreased compared with that of the estrus group mouse tail skin, suggesting that estrogen might play a role in promoting keratinocyte proliferation. Immunohistochemical staining of PCNA revealed significantly increased positive staining in epidermal cells in the estrus mouse tail epidermis compared with the ovariectomized mouse tail epidermis (Fig. 2B). PCNA expression in the skin of the mice at different stages of the estrous cycle was also examined by Western blot analysis. In agreement with the immunohistochemical

\section{KARGER}




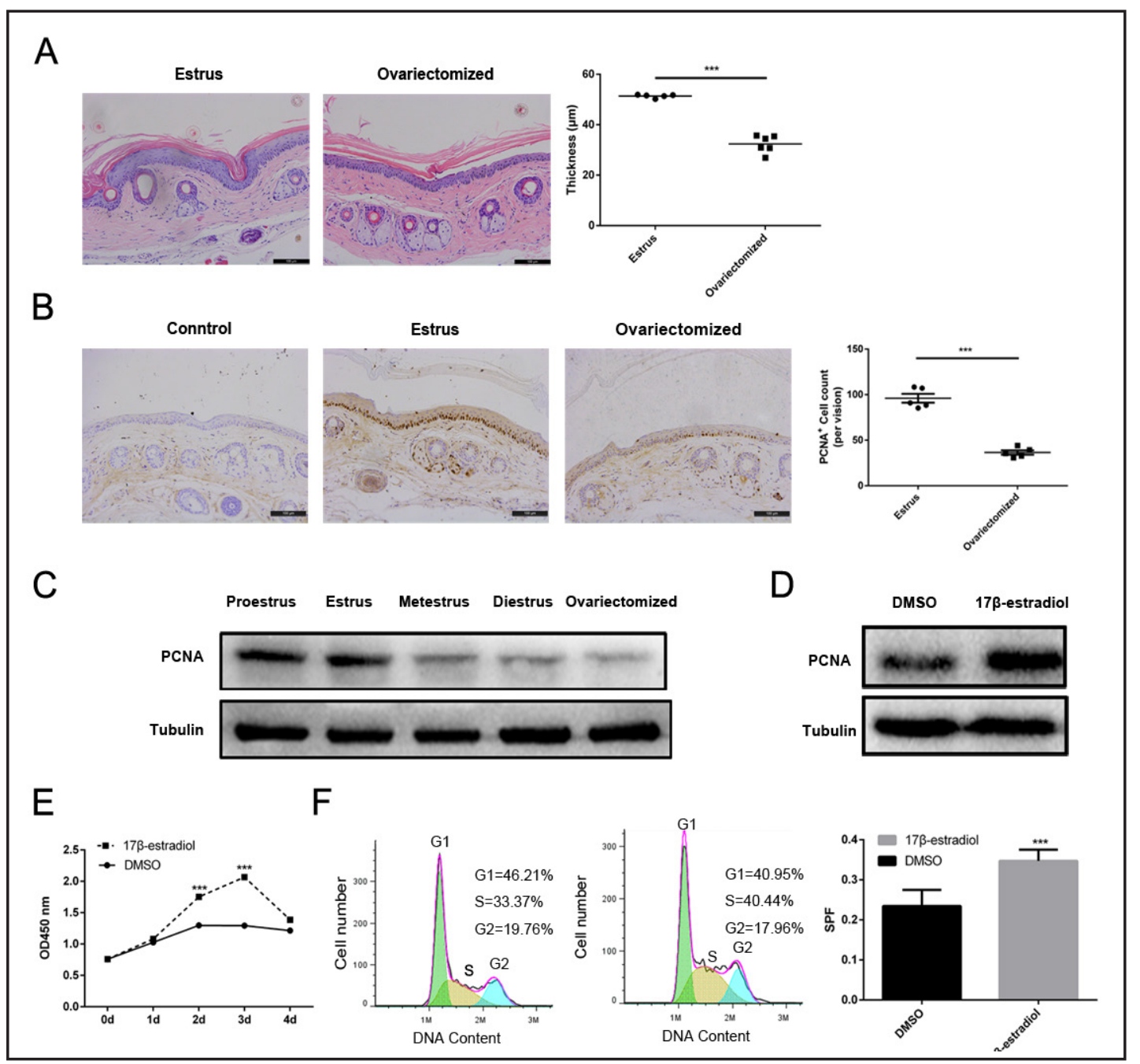

Fig. 2. Increased proliferation of keratinocytes in the presence of estrogen in vivo and in vitro. (A) HE staining and statistical analysis of tail epidermal thickness showed a thicker epidermis in the estrus mice compared with the ovariectomized mice ( $<$ 0.001). (B) Immunohistochemistry and statistical analyses showed the presence of significantly more $\mathrm{PCNA}^{+}$cells in the epidermises of the estrus mice compared with those of the ovariectomized mice $(\mathrm{p}<0.001)$. (C) Western blotting results showing the increased PCNA level in the estrus mice compared with that in the ovariectomized mice. (D) Western blotting results showing the increased PCNA level in HaCaT cells of the $17 \beta$-estradiol group compared with that of the control group. (E) CCK8 assay of HaCaT cells showing the higher proliferation rate for the estrus mice than the ovariectomized mice at different time points after treatment $\left({ }^{* *} \mathrm{p}<0.0001\right)$. (F) Representative histograms of flow cytometry analysis and quantification results showing the higher SPF of the $17 \beta$-estradiol group (treated for $72 \mathrm{~h}$ ) than the control group $\left({ }^{* * *} \mathrm{p}<0.0001\right)$. All data were calculated and are presented as the mean \pm SD of four independent experiments.

staining results, significantly higher PCNA expression levels were observed in the skin of the mice at proestrus and estrus phases compared with the skin of those at metestrus and diestrus phases, whereas the lowest PCNA expression level was observed in the skin of the ovariectomized mice (Fig. 2C). Thus, PCNA expression in the skin was positively correlated with the estrogen level. Furthermore, Western blotting demonstrated that PCNA expression was upregulated in $17 \beta$-estradiol-treated HaCaT cells $(100 \mathrm{nM}$ for all related experiments, Fig. 2D). To validate the effect of $17 \beta$-estradiol on promoting keratinocyte proliferation in vitro, CCK8 assay was employed. As shown in Fig. 2E, a significant elevation in HaCaT

\section{KARGER}




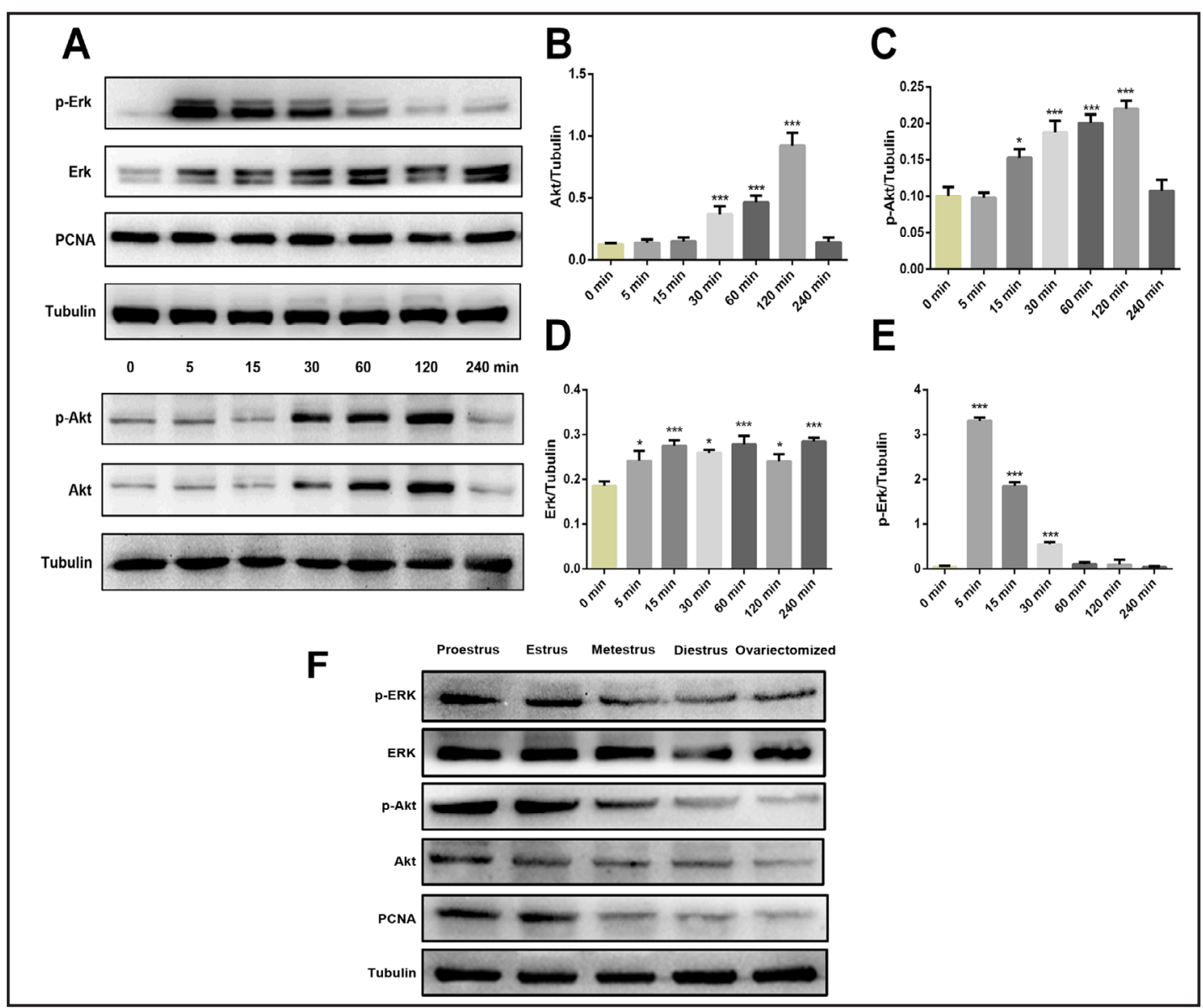

Fig. 3. Activation of the Erk/Akt signaling pathway is induced by $17 \beta$-estradiol in vitro and in vivo. (A) Western blot analysis of the targeted proteins showing the expression of Akt, p-Akt, Erk, and p-Erk at different time points of $17 \beta$-estradiol treatment. The corresponding statistical analysis results are shown in B-E as histograms ( $\left.{ }^{* * *} \mathrm{p}<0.0001\right)$. (F) Western blot analysis showing increased Akt, p-Akt, Erk, p-Erk and PCNA levels in the estrous mice compared with the ovariectomized mice.

cell proliferation was detected following treatment with $17 \beta$-estradiol compared with the vehicle control. Flow cytometry analysis revealed that a majority of the DMSO-treated cells were at the G1-S phase of the cell cycle, whereas most of the $17 \beta$-estradiol-treated HaCaT cells were at the S phase (Fig. 2F). Calculation of the SPF indicated that the number of $S$ phase cells in the $17 \beta$-estradiol-treated group was significantly increased compared with that in the DMSO group (Fig. 2F). These in vivo and in vitro results demonstrate that estrogen promotes keratinocyte proliferation with the subsequent upregulation of PCNA expression.

\section{ERK and Akt signaling pathway were activated by $17 \beta$-estradiol}

To determine the effects of $17 \beta$-estradiol on keratinocyte intracellular signaling pathways, HaCaT cells were maintained under previously described conditions [23]. 17 $\beta$-Estradiol was added to the medium, and the Akt and Erk protein levels and phosphorylation statuses were determined (Fig. 3A). During the first $240 \mathrm{~min}$, significant upregulation of both Akt and Erk was detected at 120 and $60 \mathrm{~min}$, respectively (Fig. 3B, D). Moreover, the phosphorylation of Akt and Erk was maximally stimulated above the baseline levels at 60 120 and 5-15 min, respectively (Fig. 3C, E), indicating that Erk activation occurred much earlier than Akt activation. Notably, the activation of both Akt and Erk was subsequently attenuated (Fig. 3A-E). No obvious upregulation of the PCNA protein level was detected during the first $240 \mathrm{~min}$ of $17 \beta$-estradiol stimulation (Fig. 3A).

\section{KARGER}




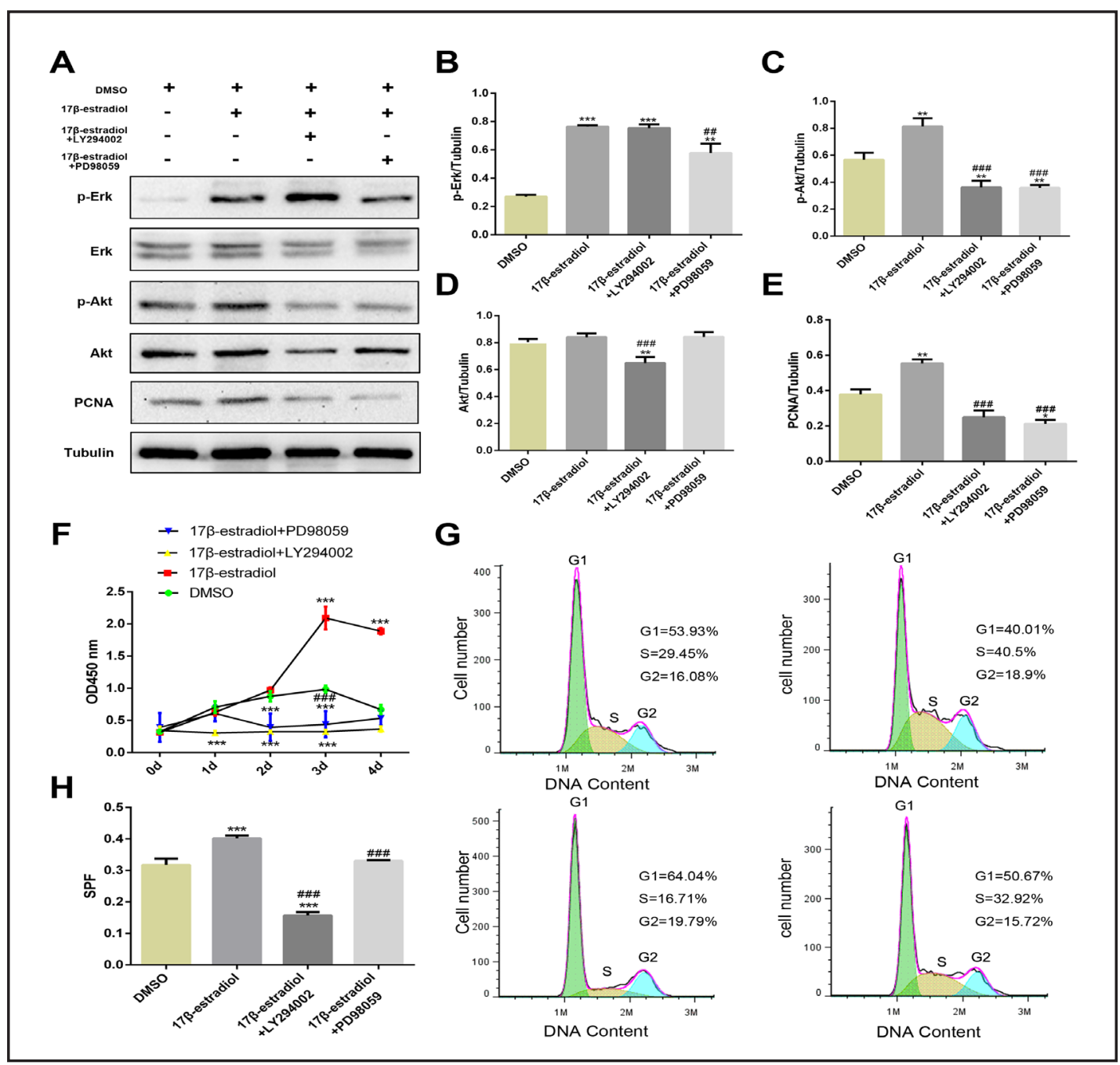

Fig. 4. Inhibition of the Erk or Akt signaling pathway reversed $17 \beta$-estradiol-induced HaCaT cell proliferation. (A) Western blot analysis showing higher PCNA, Akt, p-Akt, Erk and p-Erk expression in the $17 \beta$-estradiol group than in the other three groups. (B-E) The corresponding statistical analysis results for the Western blot are shown on the right sides of the histograms. The data were obtained from three independent experiments. (F) CCK8 assay showing the higher HaCaT cell proliferation rate in the $17 \beta$-estradiol group than in the other three groups at each time point after treatment, while those in the Akt inhibitor-LY294002 group and Erk inhibitor-PD98059 group were significantly decreased compared with that in the DMSO group. (G) Representative histograms for flow cytometry analysis showing the higher SPF in the estrus group than in the three other groups. $(\mathrm{H})$ The corresponding statistical analysis results for flow cytometry are presented in the histograms. The data were obtained from four independent experiments. ${ }^{* * *} \mathrm{p}<0.0001$ compared with the DMSO group, and ${ }^{\# \# ~} \mathrm{p}<0.0001$ compared with the $17 \beta$-estradiol group.

Furthermore, we measured the expression of p-Erk, Erk, p-Akt, Akt and PCNA in the skin of the mice at different phases of estrous. The levels of Akt, p-Akt, and p-Erk were markedly increased in the skin of the mice at proestrus and estrus phases compared with those at metestrus and diestrus phases and the ovariectomized mice (Fig. 3F).

$17 \beta$-Estradiol promotes keratinocyte proliferation by activating the Erk/Akt/PCNA signaling pathway in HaCaT cells

Western blot analysis indicated that the increased expression of PCNA induced by 17 $\beta$-estradiol could be remarkably reversed by a specific inhibitor of Erk (PD98059) or of Akt 


\section{Cellular Physiology Cell Physiol Biochem 2016;38:959-968 \\ and Biochemistry Published online: March 04, $2016 \quad$\begin{tabular}{l|l} 
DOI: 10.1159/000443048 & $\begin{array}{l}\text { (c) } 2016 \text { The Author(s). Published by S. Karger AG, Basel } \\
\text { www.karger.com/cpb }\end{array}$
\end{tabular} \\ Zhou et al.: Estrogen Promotes Keratinocyte Proliferation}

(LY294002), further demonstrating the involvement of the Erk and Akt signaling pathways in this physiological context (Fig. 4A). In addition, the p-Akt levels were significantly attenuated following supplementation with the Erk inhibitor PD98059, whereas the p-Erk levels showed no difference after treatment with the Akt inhibitor LY294002 (Fig. 4B, C), suggesting that Erk activation might be the upstream event of estrogen-mediated wound healing. We next performed CCK8 and flow cytometry assays to determine whether inhibition of the Akt or Erk signaling pathway prevents $17 \beta$-estradiol-induced HaCaT proliferation. As expected, the HaCaT cells exhibited increased proliferation after treatment with $17 \beta$-estradiol compared with those treated with DMSO. This increase was significantly reversed after administration of an Akt or Erk inhibitor (Fig. 4F). Flow cytometry assay showed similar results, further indicating that Erk and Akt mediated $17 \beta$-estradiol-induced HaCaT proliferation (Fig. 4G, $\mathrm{H})$.

\section{Discussion}

An accumulating body of evidence has demonstrated that estrogen plays an important role in cutaneous wound healing. A previous case-cohort study of venous ulceration and pressure ulcers in elderly women has reported that patients receiving estrogen replacement are less likely to develop venous ulceration than those not receiving estrogen replacement [13]. A comparison of wound healing in pre- and post-menopausal women has demonstrated delayed re-epithelialization in the post-menopausal women, which was reversed in those administered replacement estrogen [30]. In addition, the systemic and topical application of estrogen has been shown to promote cutaneous wound healing both in vivo and in vitro $[17,30,31]$. In this study, the ovariectomized mice displayed significantly delayed cutaneous wound healing compared with the mice with a normal estrous cycle.

Estrogen plays a prominent role in accelerating epidermal keratinocyte proliferation $[16,32]$. In women with a low estrogen level, skin thickness is reduced by greater than $1 \%$ per year after menopause [16]. A study of topical estrogen administration in elderly males and females has demonstrated that keratinocyte proliferation and epidermal thickness increase significantly after only two weeks [17]. In this study, mouse tail skin was used because of the multiple layers of epidermis in the tail skin, which resembles human skin morphology [31]. A significant decrease in the epidermal thickness of the tail skin was observed in the estrogen-deficient ovariectomized mice compared with the mice with a normal estrous cycle. Moreover, the PCNA levels in the ovariectomized mice were markedly reduced compared with those in the estrus group mice, indicating less vigorous proliferation of the epidermis in the ovariectomized mice.

The Erk and Akt signaling pathways, which are known to be crucial in cutaneous wound healing [22, 33], can be modulated by numerous agents $[18,19,21]$, including $17 \beta$-estradiol [23]. Moreover, their roles in promoting cell proliferation have been reported. For instance, activation of estrogen receptor by Akt and Erk mediated estrogen receptor has been reported to promote breast cancer cell proliferation [34]. Furthermore, Verdier-Sevrain et al. have reported that the Erk signaling pathway is activated through non-genomic pathways in the physiological process of exogenous estrogen-induced keratinocyte proliferation [23]. Therefore, we hypothesize that estrogen accelerates cutaneous wound healing by promoting proliferation of epidermal keratinocytes via the Erk/Akt signaling pathway.

As reported in previous studies, $17 \beta$-estradiol induces Akt and ERK1/2 phosphorylation in a time-dependent manner, which has been shown to fluctuate in rat cortical neuron cells [35]. This phenomenon is consistent with our observation that the activation of Erk and Akt began within 5 to 60 min of estrogen treatment and was downregulated thereafter. Erk and Akt activation could still be detected on day 3 in HaCaT cells treated with $17 \beta$-estradiol in vivo, demonstrating that the degree of Erk and Akt activation was positively correlated with the estrogen level. However, PCNA expression was not upregulated concurrent with Erk or Akt expression until 3 days after $17 \beta$-estradiol treatment (after Erk/Akt expression 


\section{Cellular Physiology Cell Physiol Biochem 2016;38:959-968

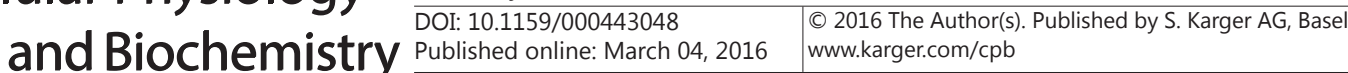 \\ Zhou et al.: Estrogen Promotes Keratinocyte Proliferation}

was altered), and it was inhibited by the Akt and Erk inhibitors, suggesting that PCNA upregulation is a downstream event. Western blotting of skin tissues obtained at different phases of the estrous cycle demonstrated in vivo fluctuations of Erk/Akt activation and PCNA expression.

Moreover, the in vitro study revealed that the proliferation rate of keratinocytes was increased following administration of $17 \beta$-estradiol and that it was halted by inhibition of Akt and Erk activities with the inhibitors LY294002 and PD98059, respectively, even when $17 \beta$-estradiol was also present, demonstrating a clear relationship between estrogen and the Erk/Akt signaling pathways in mediating keratinocyte proliferation. In addition, we discovered that Erk was upregulated and activated at the beginning of the process, whereas Akt was upregulated subsequently. Furthermore, the p-Akt level was significantly attenuated by supplementation with the Erk inhibitor PD98059, whereas the p-Erk level was not influenced by treatment with the Akt inhibitor LY294002. These results indicate that 17 $\beta$-estradiol-induced keratinocyte proliferation may be mediated by the Akt/Erk signaling pathway.

Collectively, this study has demonstrated that estrogen accelerates cutaneous wound healing by promoting epidermal keratinocyte proliferation, involving Erk as an upstream molecular and Akt as a downstream molecular. Notably, cutaneous wound healing is an extremely complicated pathophysiological process that involves a series of important events other than keratinocyte proliferation, including inflammation and re-epithelialization. Estrogen may affect these events, and further investigation is necessary.

\section{Acknowledgements}

This work was supported by a grant from the State Key Laboratory of Trauma, Burns and Combined Injury of China (No. SKLZZ201230), the National Natural Science Foundation of China (No. 81201464), and the Specific Project of Health, Ministry of Health of China (No. 201202002) and by a grant from the CPLA Scientific Research Fund (No. BWS11J039).

\section{Disclosure Statement}

The authors of this article declare that there are no conflicts of interest.

\section{References}

1 Singer AJ, Clark RA: Cutaneous wound healing. New Engl J Med. 1999;341:738-746.

2 Gurtner GC, Werner S, Barrandon Y, Longaker MT: Wound repair and regeneration. Nature 2008;453:314321.

3 Borena BM, Martens A, Broeckx SY, Meyer E, Chiers K, Duchateau L, Spaas JH: Regenerative Skin Wound Healing in Mammals: State-of-the-Art on Growth Factor and Stem Cell Based Treatments. Cell Physiol Biochem 2015;36:1-23.

4 Hopkinson SB, Hamill KJ, Wu Y, Eisenberg JL, Hiroyasu S, Jones JC: Focal contact and hemidesmosomal proteins in keratinocyte migration and wound repair. Adv Wound Care (New Rochelle) 2014;3:247-263.

5 Yang CT, Zhao Y, Xian M, Li JH, Dong Q, Bai HB, Xu JD, Zhang MF: A novel controllable hydrogen sulfidereleasing molecule protects human skin keratinocytes against methylglyoxal-induced injury and dysfunction. Cell Physiol Biochem 2014;34:1304-1317.

6 Werner S, Krieg T, Smola H: Keratinocyte-fibroblast interactions in wound healing. J Invest Dermatol 2007;127:998-1008.

7 Bielefeld KA, Amini-Nik S, Alman BA: Cutaneous wound healing: recruiting developmental pathways for regeneration. Cell Mol Life Sci 2013;70:2059-2081.

8 Wadman M: Scar prevention: The healing touch. Nature 2005;436:1079-1080.

9 Martinotti S, Ranzato E: Propolis: a new frontier for wound healing? Burns Trauma 2015;3:1-7.

10 Lien L, Tho N, Ha D, Hang P, Nghia P, Thang N: Influence of phytochemicals in piper betle linn leaf extract on wound healing. Burns Trauma 2015;3:1-8. 


\section{Cellular Physiology Cell Physiol Biochem 2016;38:959-968

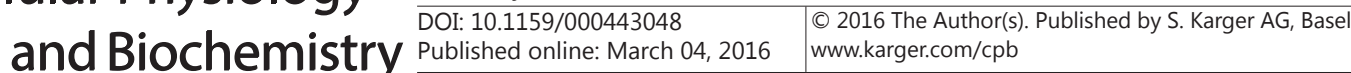 \\ Zhou et al.: Estrogen Promotes Keratinocyte Proliferation}

11 Ke T, Yang M, Mao D, Zhu M, Che Y, Kong D, Li C: Co-Transplantation of Skin-Derived Precursors and Collagen Sponge Facilitates Diabetic Wound Healing by Promoting Local Vascular Regeneration. Cell Physiol Biochem 2015;37:1725-1737.

12 Maheux R, Naud F, Rioux M, Grenier R, Lemay A, Guy J, Langevin M: A randomized, double-blind, placebo-controlled study on the effect of conjugated estrogens on skin thickness. Am J Obstet Gynecol 1994;170:642-649.

13 Margolis DJ, Knauss J, Bilker W: Hormone replacement therapy and prevention of pressure ulcers and venous leg ulcers. Lancet 2002;359:675-677.

14 Emmerson E, Hardman MJ: The role of estrogen deficiency in skin ageing and wound healing. Biogerontology 2012;13:3-20.

15 Thornton MJ: Estrogens and aging skin. Dermatoendocrinol 2013;5:264-270.

16 Brincat M, Versi E, Moniz CF, Magos A, de Trafford J, Studd JW: Skin collagen changes in postmenopausal women receiving different regimens of estrogen therapy. Obstet Gynecol 1987;70:123-127.

17 Son ED, Lee JY, Lee S, Kim MS, Lee BG, Chang IS, Chung JH: Topical application of 17beta-estradiol increases extracellular matrix protein synthesis by stimulating tgf-Beta signaling in aged human skin in vivo. J Invest Dermatol 2005;124:1149-1161.

18 Chen JC, Lin BB, Hu HW, Lin C, Jin WY, Zhang FB, Zhu YA, Lu CJ, Wei XJ, Chen RJ: NGF accelerates cutaneous wound healing by promoting the migration of dermal fibroblasts via the PI3K/Akt-Rac1-JNK and ERK pathways. Biomed Res Int 2014;2014:547187.

19 Watson A, Morris VL, Chan BM: Coordinated integrin and growth factor regulation of primary keratinocyte migration mediated through extracellular signal regulated kinase and phosphoinositide 3-kinase. Arch Dermatol Res 2009;301:307-317.

20 Ouyang W, Li J, Zhang D, Jiang BH, Huang DC: PI-3K/Akt signal pathway plays a crucial role in arseniteinduced cell proliferation of human keratinocytes through induction of cyclin D1. J Cell Biochem 2007;101:969-978.

21 Lee SH, Zahoor M, Hwang JK, Min do S, Choi KY: Valproic acid induces cutaneous wound healing in vivo and enhances keratinocyte motility. PLoS One 2012;7:e48791.

22 Weber TJ, Shankaran H, Wiley HS, Opresko LK, Chrisler WB, Quesenberry RD: Basic fibroblast growth factor regulates persistent ERK oscillations in premalignant but not malignant JB6 cells. J Invest Dermatol 2010;130:1444-1456.

23 Verdier-Sevrain S, Yaar M, Cantatore J, Traish A, Gilchrest BA: Estradiol induces proliferation of keratinocytes via a receptor mediated mechanism. FASEB J 2004;18:1252-1254.

24 Marcondes FK, Bianchi FJ, Tanno AP: Determination of the estrous cycle phases of rats: some helpful considerations. Braz J Biol 2002;62:609-614.

25 Macmillan KL, Burke CR: Effects of oestrous cycle control on reproductive efficiency. Anim Reprod Sci 1996;42:307-320.

26 Goldman JM, Murr AS, Cooper RL: The rodent estrous cycle: characterization of vaginal cytology and its utility in toxicological studies. Birth Defects Res B Dev Reprod Toxicol 2007;80:84-97.

27 Koba S, Yoshinaga K, Fujita S, Miyoshi M, Watanabe T: Exercise pressor reflex function in female rats fluctuates with the estrous cycle. J Appl Physiol (1985) 2012;113:719-726.

28 Xiong H, Xu Y, Tan G, Han Y, Tang Z, Xu W, Zeng F, Guo Q: Glycyrrhizin ameliorates imiquimod-induced psoriasis-like skin lesions in BALB/c mice and inhibits TNF-alpha-induced ICAM-1 expression via NFkappaB/MAPK in HaCaT cells. Cell Physiol Biochem 2015;35:1335-1346.

29 Akgul B, Ghali L, Davies D, Pfister H, Leigh IM, Storey A: HPV8 early genes modulate differentiation and cell cycle of primary human adult keratinocytes. Exp Dermatol 2007;16:590-599.

30 Ashcroft GS, Dodsworth J, van Boxtel E, Tarnuzzer RW, Horan MA, Schultz GS, Ferguson MW: Estrogen accelerates cutaneous wound healing associated with an increase in TGF-beta1 levels. Nat Med 1997;3:1209-1215.

31 Ashcroft GS, Greenwell-Wild T, Horan MA, Wahl SM, Ferguson MW: Topical estrogen accelerates cutaneous wound healing in aged humans associated with an altered inflammatory response. Am J Pathol 1999;155:1137-1146.

32 Martin P: Wound healing_-aiming for perfect skin regeneration. Science 1997;4:75-81.

33 Squarize CH, Castilho RM, Bugge TH, Gutkind JS: Accelerated wound healing by mTOR activation in genetically defined mouse models. PLoS One 2010;5:e10643.

34 Wei Z, Song X, Shaikh ZA: Cadmium promotes the proliferation of triple-negative breast cancer cells through EGFR-mediated cell cycle regulation. Toxicol Appl Pharmacol 2015;289:98-108.

35 Mannella P, Brinton RD: Estrogen receptor protein interaction with phosphatidylinositol 3-kinase leads to activation of phosphorylated Akt and extracellular signal-regulated kinase 1/2 in the same population of cortical neurons: a unified mechanism of estrogen action. J Neurosci 2006;26:9439-9447. 